\title{
MORPHING AIRFOILS ANALYSIS USING DYNAMIC MESHING
}

\author{
Chawki Abdessemed ${ }^{1}$, Yufeng $\mathrm{Yao}^{2}$, Abdessalem Bouferrouk ${ }^{3}$, Pritesh Narayan ${ }^{4}$ \\ Department of Engineering Design and Mathematics, University of the West of England, Coldharbour Lane, \\ Frenchay, Bristol, BS16 1QY, United Kingdom.
}

\begin{abstract}
Purpose -The purpose of this paper is to use dynamic meshing to perform CFD analyses of a NACA 0012 airfoil fitted with a morphing trailing-edge (TE) flap when it undergoes static and time dependent morphing. The steady CFD predictions of the original and morphing airfoils are validated against published data. The study also investigates an airfoil with a hinged trailing edge flap for aerodynamic performance comparison. The study further extends to an unsteady CFD analysis of a dynamically morphing trailing edge flap for proof-of-concept and also to realise its potential for future applications.

Design/methodology/approach -An existing parametrization method was modified and implemented in a user-defined function (UDF) to perform dynamic meshing which is essential for morphing airfoil unsteady simulations. The results from the deformed mesh were verified to ensure the validity of the adopted mesh deformation method. ANSYS Fluent software was used to perform steady and unsteady analysis and the results were compared with computational predictions.

Findings -Steady computational results are in good agreement with those from OpenFOAM for a non-morphing airfoil, and for a morphed airfoil with a maximum TE deflection equal to $5 \%$ of the chord. The results obtained by ANSYS Fluent show that an average of $6.5 \%$ increase in lift-to-drag ratio is achieved, compared with a hinged flap airfoil with the same TE deflection. By using dynamic meshing, unsteady transient simulations reveal that the local flow field is influenced by the morphing motion.

Originality/value -An airfoil parametrization method was modified to introduce time dependent morphing and used to drive dynamic meshing through an in-house developed UDF. The morphed airfoil's superior aerodynamic performance was demonstrated in comparison with traditional hinged trailing edge flap. A methodology was developed to perform unsteady transient analysis of a morphing airfoil at high angles of attack beyond stall, and to compare with published data. Unsteady predictions have shown signs of rich flow features, paving the way for further research into the effects of a dynamic flap on the flow physics.
\end{abstract}

Keywords Morphing airfoil, Hinged flap, Dynamic meshing, Computational Fluid Dynamics, Dynamic morphing, Steady and Unsteady Flow Analysis.

Paper type Research paper

\section{Introduction}

Morphing wing is a bio-inspired concept that offers the aircraft the capability to change its aerodynamic characteristics during its flight mission. Compared with a fixed (rigid) wing configuration that is normally optimised for a particular

\footnotetext{
${ }^{1} \mathrm{PhD}$ student. Chawki. Abdessemed@uwe.ac.uk

${ }^{2}$ Professor.Yufeng.Yao@uwe.ac.uk

${ }^{3}$ Senior lecturer. Abdessalem.Bouferrouk@uwe.ac.uk

${ }^{4}$ Senior lecturer. Pritesh.Narayan@uwe.ac.uk
} 
design point and hence, optimised for a fixed set of parameters (e.g. altitude, Mach number, weight), morphing concepts can be continuously deflected in-flight to produce optimal performance throughout the entire flight envelope. Barbarino et al. (2011) provided a thorough review of the progress made in morphing concepts and the possible benefits that can be gained using this technology. It was noted that if a $1 \%$ drag reduction could be obtained with morphing concepts, $\$ 140$ million/year could be saved on fuel necessary to operate the US aircraft fleet. However, the paper also pointed out that the added complexity and weight issue must be addressed in order for this technology to mature and be flight-ready. Morphing technology can contribute to the increase in the aerodynamic efficiency due to the optimised lift-to-drag ratio (see e.g. the works of Hilbig and Wagner, 1984; Urnes and Nguyen, 2013), which would lead to an extended cruise range and reduction of specific fuel consumption. A decrease in aerodynamic noise is also possible using morphing technologies as demonstrated by Ai et al. (2016b) who showed that a NACA 63-418 airfoil fitted with a morphing trailing edge flap can offer a noise reduction of up to $3 \mathrm{~dB}$. A more drastic approach for noise reduction surveyed by Dobrzynski, (2010) is filling the gaps formed by traditional discrete surfaces to effectively eliminate the flaps side-edges. This way, an aircraft can have smoother structural surfaces when using a morphing device such as a trailing edge flap, which conforms to the wing surface. An example of this approach is the elastically lofted transition concept aiming at filling the gaps produced by a morphing flap as presented by Woods et al. (2016). Furthermore, morphing concepts are not confined only to rigid aircraft wings; recent years have seen an increasing trend in morphing concepts applied to rotary wing configurations. For instance, in the review by Lachenal et al. (2013) it was noted that morphing helicopter blades were proven to be key for load alleviation, and that for wind turbine blades, morphing trailing edge flaps can offer higher efficiency.

Nevertheless, morphing structures introduce their own challenges to the design and analysis process. First, new adaptive materials need to be integrated for morphing application in order to counter balance the added weight for actuations. Second, advanced control and optimization algorithms should be developed to provide the most advantageous improvements from a morphing configuration. Last but not least, there is a need for accurate predictions of the unsteady forces (lift, drag) and moments acting on dynamic morphing configurations in order to estimate the possible efficiency improvements, or declines, due to a morphing concept. The latter point will be addressed in this paper.

In the past, various numerical methods were used to analyse morphing wing performance. For example, the use of steady and unsteady vortex-lattice methods (VLM) was popular for morphing configurations since they offered better accuracy than analytical methods or strip line theory, while still consuming less CPU time than the full Navier-Stokes simulations. For instance, VLM was used to model a morphing Gull wing (Obradovic and Subbarao, 2012), to predict and optimise the lift-to-drag ratio of a variable camber morphing wing (Urnes and Nguyen, 2013), and to compare the performance of various morphing wing concepts that have optimised camber and span (Molinari et al., 2011). Later, a doublet-lattice method (DLM) corrected by high accuracy CFD data was developed and used to model a morphing wing tip concept (Chekkal et al., 2015). Most recent aerodynamic optimization problems have typically used an aerodynamic model based on the Reynolds Averaged Navier-Stokes (RANS) equations coupled with turbulence models. For instance, such technique was applied to model both a morphing trailing edge (TE) and a full morphing wing (Lyu and Martins, 2014), where it was found that a drag reduction of 1-5\% was possible, depending on flight phase and conditions.

Furthermore, the use of morphing trailing edge flaps to improve the aerodynamic performance of airfoils has recently been investigated. Wolff et al. (2014) used steady and unsteady RANS to analyse the performance of a thin wind turbine airfoil fitted with actively morphing trailing edges. The dynamic trailing edge deflections were simulated with the help of structured multi-block deformable meshes within the code FLOWer (Kroll and Fassbender, 2002). The authors discovered that such morphing trailing edge sections can have a significant impact on the lift coefficient and stall behaviour of the wind turbine. However, it is necessary to extend dynamic morphing in CFD for typical wing sections beyond thin airfoils used for wind turbines. A steady flow analysis of a morphing airfoil concept was presented in the work of Woods et al., (2014a) where the RANS based solver OpenFOAM (Jasak et al., 2007) was used to simulate the flow around various deflected configurations, in addition to a comparative study with the panel code XFOIL (Drela, 1989). It was found that the two codes produced good agreement up to $8^{\circ}$ angle of attack (AoA), and it was observed that the closer the starting morphing location was to the leading edge, the more drag reduction that could be achieved. Ai et al. (2016a) studied, both experimentally and numerically, the aerodynamic performance of the NACA 0012 airfoil when fitted with morphing trailing edges. It was established that the improvement in aerodynamic performance was dependent on the amount of camber introduced on the trailing edge. The amount of camber also controlled the level of flow separation delay near the trailing edge at high angles of attack. However, the numerical simulations (using OpenFOAM with Spalart-Allmaras turbulence model) were limited to AoA before stall and only steady state RANS were used to validate their experimental work on static deflections.

The focus of this paper will be on the use of dynamic meshing to model two morphing cases for the NACA 0012 airfoil. The first case is a steady flow analysis of a trailing edge section when it is statically deflected. The second case deals with an unsteady flow analysis when the trailing edge is morphed continuously (in time) from its baseline geometry 
to a maximum deflection position, hereby called unsteady or dynamic morphing. A mathematical model based on a modified version of the method developed by Woods et al. (2013) will be adopted to parametrize the geometry and its deformations. The parametrization technique will be subsequently implemented in a user-defined function (UDF) used to control the dynamic meshing schemes available in the commercial solver ANSYS Fluent. A numerical validation of the steady state findings against published results will be performed and the aerodynamic efficiency of the morphing concept will be compared to an airfoil with a hinged trailing-edge flap. In order to complement the steady state analysis, a method of performing unsteady flow analysis of airfoils with a dynamic morphing trailing edge will be developed to demonstrate its capability of simulating a deformable geometry, and to analyse the resulting flow behaviour.

\section{Methodology}

\subsection{Unsteady geometry parameterization}

Most morphing airfoil studies often parametrize the deformation only in a steady, static manner. However, in order to perform simulations during a dynamic geometry deformation process, it is essential to introduce time into the parametrization process to allow an unsteady CFD analysis to produce results closer to real life morphing. In this paper, the parametrization of geometry will be based on the Fish Bone Active Camber (FishBAC) concept (Woods et al., 2014b) which is then modified for unsteady morphing. In its original implementation, the method adopted a relatively simple approach to model a NACA 0012 wing undergoing trailing edge deflections, starting at different chord stations and for various maximum deflections. The baseline airfoil was morphed by modifying the camber definition of the targeted morphing region of the chord, followed by reconstruction of a new airfoil shape using some control parameters. The morphing starts with the definition of a parameter $x_{s}$ and the airfoil shape is built up by the accumulation of the baseline NACA 0012 thickness distribution and a parametrically defined camber line. The NACA four series thickness distribution is defined in Eq. 1 (Jacobs et al., 1933):

$$
y_{t}=\left(\frac{t h}{0.2}\right)\left(0.2969 \sqrt{\bar{x}}-0.1260 \bar{x}-0.3516 \bar{x}^{2}+0.2843 \bar{x}^{3}-0.1015 \bar{x}^{4}\right)
$$

where $y_{t}=\frac{y}{c}$ is the non-dimensional thickness distribution, $\bar{x}=\frac{x}{c}$ is the non-dimensional distance along the chord $c$, and th is the maximum thickness as a fraction of the chord (e.g. $t h=0.12$ for a NACA 0012 section).

A third-order polynomial function (Eq. 2) was used to define the camber line ( $y_{\mathrm{c}}$ ) of the morphing part of the airfoil, and was parametrized to have a direct control over the trailing edge maximum deflection:

$$
y_{c}=\left\{\begin{array}{c}
0,0 \leq \bar{x}<x_{s} \\
-w_{t e} \cdot\left(\bar{x}-x_{s}\right)^{3} /\left(1-x_{s}\right)^{3}, \bar{x} \geq x_{s}
\end{array}\right.
$$

where $w_{t e}$ is the value of maximum deflection at the trailing edge, and $x_{s}$ is the start location for the morphing. The thickness distribution (Eq. 1) is then added to the camber distribution (Eq. 2) to get the upper and lower surface coordinates of the morphed airfoil. In the present work, this static parametrization has been extended to include a time dependency, which makes it possible to introduce unsteady dynamic morphing motion as seen in Eq. 3: 


$$
y_{c}=\left\{\begin{array}{l}
0 \quad, 0 \leq \bar{x}<x_{s} \text { and } 0 \leq t \leq \frac{T}{4} \\
\frac{-w_{t e} \sin \left(\frac{2 \pi t}{T}\right)\left(\bar{x}-x_{s}\right)^{3}}{\left(1-x_{s}\right)^{3}}, \bar{x} \geq x_{s} \text { and } 0 \leq t \leq \frac{T}{4} \\
\frac{-w_{t e}\left(\bar{x}-x_{s}\right)^{3}}{\left(1-x_{s}\right)^{3}}, \bar{x} \geq x_{s} \text { and } t>\frac{T}{4}
\end{array}\right.
$$

where $t$ is time and $T$ is the complete period of the airfoil's trailing-edge motion. The description in Eq. 3 is specifically modified so that the trailing edge can be deflected from the baseline geometry (starting $t=0 \mathrm{~s}$ ) to one with the maximum deflection $w_{t e}$ in a morphing time interval $t_{m o r p h}=\frac{T}{4}$, giving direct control over the speed of the trailing-edge deflection. At $t_{\text {morph }}=\frac{T}{4}$ the morphing stops and the geometry is fixed at the maximum deflection $w_{t e}$ therefore for our application only the motion achieved in a quarter of a period $T$ is simulated. It is worth mentioning that this formulation will be implemented in a UDF to drive the dynamic meshing scheme available in ANSYS Fluent.

\subsection{Dynamic meshing and CFD solver}

Flow simulation around a morphing wing configuration involves moving boundaries. In order to get an accurate solution of the unsteady flow computation, reliable methods for moving the computational grids are necessary since mesh re-generation would be time consuming and computationally expensive. To this end, various dynamic meshing techniques had previously been developed such as the Transfinite Interpolation for structured mesh (Wang, 1994), and connectivity based methods for unstructured mesh (Rausch et al., 1989). An example of commercial software that takes advantage of these dynamic meshing capabilities is ANSYS Fluent which is used in this paper. The main advantage about Fluent dynamic meshing is that the user only needs to create a single mesh which does not need to be re-generated when dynamic motion sets in; it simply follows the deformation. A different approach was used in the study of Wolff et al. (2014) where the authors used a deformable mesh in their FLOWer code using a fully structured mesh, but several meshes had to be created for various deflections. Linear interpolation was used each time a new mesh was read into the solver, which is quite cumbersome and time consuming compared to simple mesh deformation in Fluent. In the present study, the results obtained from Fluent will also be compared to those obtained by XFOIL and OpenFOAM. XFOIL is a high-order panel method code which uses a linear-vorticity stream function formulation to solve for the flow field. In order to account for the viscous effects, a viscous boundary layer component is included for calculating skin friction drag and flow separation. OpenFOAM is a set of open source CFD tools used for high fidelity simulations, having a variety of components. The results used for comparison in this study were obtained using the simpleFOAM solver which utilises the SIMPLE algorithm to solve the incompressible RANS, $2^{\text {nd }}$ order schemes for spatial gradients limited by Sweby flux limiter for stability (Woods et al., 2014a).

Dynamic meshing in ANSYS Fluent is controlled by the bias of the development of a UDF which makes use of available macros. For example, the DEFINE_GRID_MOTION macro (Fluent Inc., 2011) was used to control each boundary node separately to enable the relative movement between adjacent nodes. Consequently, this allows the modelling of deforming, non-rigid bodies. The modified parametrization technique was implemented in a UDF to enable the use of the dynamic meshing in ANSYS Fluent for flow analysis. For deformation problems such as morphing wings, the smoothing and remeshing methods are of interest but the remeshing technique is only available for tetrahedral mesh cells; therefore, the mesh used in the present simulations was generated to take advantage of this remeshing technique in a local region around the trailing-edge, while retaining high quality structured mesh for most of the domain. Fluent's smoothing function enables mesh adjustment in those zones where deformation occurs. It permits the interior nodes to 'absorb' the deformation without any change in the number of nodes or their connectivity (i.e. topology is preserved). While diffusion-based smoothing is computationally more expensive, it tends to produce a better quality mesh particularly near the boundaries of a deforming body (ANSYS Inc., 2015) by causing the far field nodes to absorb the deformation. 
Therefore, this smoothing method was chosen for this morphing airfoil application along with local cell remeshing techniques.

\section{Problem definition and numerical setup}

The physical problem studied in this paper is based the FishBAC configuration as used by Woods et al. (2014a) and which is capable of performing large-scale camber deformations. In order to study this morphing concept in an unsteady fashion, robust mesh deformation schemes are needed. Therefore, before performing the unsteady analysis, a steady CFD analysis was conducted to verify that the deformed mesh gives similar results to a mesh generated at the same deflection angle. The FishBAC morphing concept with a maximum deflection value of $w_{t e}=0.05$ or $5 \%$ of the chord was analysed in two different ways. First, the deformed mesh case, i.e. the airfoil was deformed from the baseline NACA 0012 to the maximum deflection $w_{t e}$ (Fig. 1a) then a steady simulation was run with the resulting mesh. Second, the re-generated mesh case, i.e. a good quality mesh was re-generated around the already deflected geometry after which the steady CFD analysis was performed.

The discrepancy between the two cases was found to be less than $1 \%$ (Figure not shown) for all angles of attack, which clearly demonstrates that the mesh quality is preserved during the deformation. This is further supported by Table 1 which shows the minimum orthogonal quality for the deformed mesh to be 0.5 which is largely superior to the minimum requirement of 0.01 specified by the Fluent solver (Fluent Inc., 2011). Such preservation of the deformed mesh quality is mainly due to the fact that the highly skewed cells within the triangular patch around the airfoil's TE are systematically remeshed until the skewness or size criteria are met. Therefore, the deformed mesh was used as the default for the remainder of the study.

In addition, steady CFD analysis was performed around a hinged flap (Fig. 1b) and a morphed flap NACA 0012 airfoil configuration (Fig. 1a) to quantify the aerodynamic differences between the two. The results of the morphed case were also compared with those of Woods et al. (2014a).

Table 1. Mesh characteristics.

\begin{tabular}{cccc}
\hline Mesh & Cells & Orthogonal quality & Minimum Orthogonal Quality \\
\hline Baseline mesh & 58424 & 0.987 & 0.377 \\
Deformed mesh & 59120 & 0.973 & 0.500 \\
Regenerated mesh & 58108 & 0.993 & 0.183 \\
\hline
\end{tabular}

For the unsteady case, a UDF was used to continually deform the airfoil from the baseline NACA 0012 to a maximum deflection value of $w_{t e}=0.05$, using the same time step of CFD simulation.

The meshes that were generated around the airfoil were targeting a maximum near-wall first-layer grid resolution of $y^{+}=1$, an expansion ratio of 1.1 , a total of 400 grid points around airfoil surface, and a spacing of $10^{-4} \mathrm{~m}$ at both the leading and trailing edges. In addition, a set of refined meshes up to 900,000 elements were investigated and the discrepancies obtained in the lift and drag coefficients were generally around 1\%; therefore a mesh with about 58,000 elements was used as seen in Table 1 which summarises the mesh characteristics. The O-grid type mesh (Fig. 2b) around the NACA 0012 was created with the deformation in mind. It was found that when the flap is morphed, the structured mesh near the trailing edge becomes highly skewed. To overcome this constraint, an unstructured patch around the trailing edge (a small region of about $0.05 \%$ of the chord) was generated using an unstructured, triangular mesh (Fig. 2a). This way, the mesh retains its structured nature over the majority of the computational domain. Table 2 sums up the parameters used for dynamic meshing.

The $k$ - $\omega$ SST turbulence model was used given its suitability for modelling flows with separated regions, the pressure based SIMPLE algorithm was used for pressure-velocity coupling in addition to a least-square cell based discretization scheme, and a second order upwind scheme was used for the momentum and turbulence equations discretization. A pressure far-field boundary condition was imposed on the domain outer boundaries, located about 100 chord lengths around the airfoil to ensure there are no reflecting influences from boundaries. The Reynolds number based on the chord length $\left(\right.$ chord $=1 \mathrm{~m}$ ) and freestream flow conditions was $6.75 \times 10^{5}$, and the Mach number was 0.1 . In the study by Woods et al. (2014a), the airfoil chord was $0.3 \mathrm{~m}$, so in order to ensure that the exact Reynolds number and Mach number are replicated in this study the constant dynamic viscosity was modified to $6.174 \times 10^{-5} \mathrm{~kg} / \mathrm{m} . \mathrm{s}$. This allowed the use of a $1 \mathrm{~m}$ chord without further modifications to the parametrization method. Furthermore, to ensure that this change of dynamic 
viscosity has no effect on the results, the mesh created was scaled down by a factor of 0.3 (obtaining an airfoil with a chord $=0.3 \mathrm{~m}$ ). Using sea level standard conditions, both configurations gave similar results. The turbulence intensity was set to $1 \%$ with a turbulence length scale being the same as the airfoil chord. All simulations were run until both the lift and drag coefficients converged, and all residuals dropped below $1 \times 10^{-6}$.

Table 2. Dynamic meshing parameters.

\begin{tabular}{cccccc}
\hline Diffusion function & $\begin{array}{c}\text { Diffusion } \\
\text { parameter }\end{array}$ & $\begin{array}{c}\text { Remeshing } \\
\text { method }\end{array}$ & $\begin{array}{c}\text { Minimum } \\
\text { length scale } \\
(\mathbf{m})\end{array}$ & $\begin{array}{c}\text { Maximum } \\
\text { cell } \\
\text { skewness }\end{array}$ & Size remeshing interval \\
\hline Boundary distance & 1.5 & Local cell & $7.08 \times 10^{-6}$ & 0.5 & 1 \\
\hline
\end{tabular}

\section{Results and discussion}

\subsection{Steady state analysis}

\subsubsection{Comparison study}

Figure 3 presents current simulation results in comparison with those from OpenFOAM and XFOIL (Woods et al., 2014a) for the baseline NACA 0012, the 5\% chord deflected morphed airfoil and flapped airfoil. For the baseline NACA 0012, Fluent and OpenFOAM exhibit very good agreement for lift coefficient throughout the entire AoA range, whereas XFOIL tends to over-predict the lift. The drag coefficient values are also in good agreement between Fluent and OpenFOAM for angles of attack smaller than $10^{\circ}$. However, the discrepancies grow steadily at higher angles. These discrepancies between the high fidelity solvers (Fluent and OpenFOAM) and XFOIL could be attributed to the lack of turbulence modelling in XFOIL where viscous effects are simply modelled by adding a boundary layer solver to the inviscid formulation.

An interesting trend appears in the $5 \%$ chord morphed airfoil. For AoA lower than $8^{\circ}$, Fluent predicts an average of a $6 \%$ higher lift compared with OpenFOAM, and a slightly lower value compared with XFOIL. At the same time, both Fluent and OpenFOAM gave a drag estimate higher than XFOIL for all angles of attack studied; again the differences could be due to the approximation used by XFOIL to account for separation and skin friction. It is worth noting that both Fluent and OpenFOAM simulations used the $k-\omega S S T$ turbulence model, yet the implementation, constants and various options available in the solvers are likely to be different which may explain the differences observed ( $5 \%$ on average). In addition, the differences in the meshes used, and the fact that flux limiters were not used in the Fluent simulation can be added to the factors that may influence the results. Finally, Fluent lift coefficient results predicted that the maximum lift coefficient is reached at $\mathrm{AoA}=13^{\circ}$, which was not clearly shown in the previous study (Woods et al., 2014a), possibly due to the $2^{\circ}$ increments used in their simulations.

\subsubsection{Morphed vs hinged flap airfoil}

The performance of the NACA 0012 airfoil with a morphing flap was compared with the case when the airfoil was fitted with a hinged flap of similar size. Figure 3 a clearly shows a general increase in lift obtained by the morphed airfoil compared with the flapped one. However, this increase is accompanied by a drag penalty. A better indicator is the aerodynamic efficiency (i.e. lift-to-drag ratio, $C_{L} / C_{D}$ ) plotted in Figure $3 \mathrm{c}$. It is clear that the morphed airfoil provides a significant increase in efficiency up to an angle of attack of $12^{\circ}$ after which the morphed airfoil efficiency decreases steadily till $13^{\circ}$. The maximum efficiency increase of $13 \%$ was observed at $8^{\circ}$ AoA. This trend continues up to an angle of attack of $13^{\circ}$, beyond which the flapped airfoil somehow exhibits an increasingly better performance in the aft-stall regime. On average, the morphed airfoil provides a $6.5 \%$ increase in the $C_{L} / C_{D}$ efficiency. 
Figures $4 \mathrm{a}, 4 \mathrm{~b}$ and $4 \mathrm{c}$ show a comparison between the pressure coefficients of both configurations at three angles of attack: $0^{\circ}, 8^{\circ}$ and $14^{\circ}$. From these figures, it is shown that most of the differences are located around the modified area (morphed or hinged) even though there are more significant differences around the leading edge at $0^{\circ}$, presumably due to the flow being accelerated more by the morphed airfoil. The morphed airfoil exhibits a larger $C_{p}$ area around the trailing edge with a distribution similar to supercritical airfoils (Harris, 1990) given the comparable $C_{p}$ distribution between the two configurations. In Figures $4 \mathrm{a}-\mathrm{c}$, results show a sudden decrease in pressure coefficient at the location where the hinged flap starts. It is likely that this sudden decrease is related to the existence of the protruding hinge and subsequent impingement of the flow on it as this is the only difference between the two flaps. The hinge would cause the flow to take a sharp turn following the flap deflection (as suggested by Ai et al., 2016a). In comparison, however, the morphed flap seems to create an increase in pressure coefficient. Similar observations were reported by Ai et al. (2016a). Figure $4 \mathrm{~b}$ illustrates the maximum differences between the two airfoils.

At the AoA of $14^{\circ}$, there is a significant decrease in the $C_{p}$ area of the morphed flap airfoil even though it is still larger than the flapped airfoil and this may explain why the morphed airfoil produces more lift. However, the drag penalty makes the morphed airfoil efficiency drop below that of the hinged flap. Further, it was also shown by Troldborg (2005) that the performance of a hinged flap was inferior to that of a morphing flap. This behaviour is confirmed by the data produced from a wind tunnel experiment where the 3D FishBAC concept is compared with a 3D NACA 0012 hinged flap airfoil (Woods et al., 2014b). The Reynolds number of the experiment was around 400,000 (lower than the one used in the numerical simulations), despite that, the behaviour of both configurations is similar. The morphed airfoil showed better performance in the majority of angles of attack, with a steadily decreasing performance until both configurations gave similar efficiency at AoA of $13^{\circ}$.

\subsection{Unsteady morphing analysis}

Following validation of the steady CFD results, unsteady simulations are carried out next, where the airfoil trailingedge portion undergoes time-dependent morphing from its baseline position to a maximum deflection at TE, equal to 5\% of the chord. The purpose is to demonstrate proof-of-concept of the methodology presented to analyse a dynamically morphed airfoil, reveal some trends and investigate some underlying flow physics. In particular, the dynamic evolution of the lift and drag coefficients are presented and the flow field is analysed. The unsteady morphing analysis is carried out at higher $\operatorname{AoA}\left(14^{\circ}\right.$ and $\left.16^{\circ}\right)$ around the stall with the aims of: 1) investigating some rich dynamic flow features that have not been explored in previous studies, and 2) looking into the possible effects that morphing could exercise on the flow to possibly delay the stall. To the authors' knowledge, this is the first time a continuous morphing trailing-edge airfoil is being simulated using dynamic meshing in ANSYS Fluent.

For the unsteady analysis, simulations start from a converged steady state solution. Three time steps $\left(\Delta t=10^{-3} s, 10^{-4} s\right.$, $4 \times 10^{-5} s$ ) were investigated in order to ensure time step independence while 20 sub-iterations per time step were used to ensure numerical stability. Instead of the SIMPLE algorithm used for the steady case, the Coupled algorithm was used for the unsteady calculations given its more efficient and robust implementation, using a courant number of 1 . An overall flow time of 12 seconds was simulated to illustrate flow responses with respect to airfoil geometry change. For AoA $=14^{\circ}$, all three time steps gave similar results, confirming that the study is time step independent. For a time step of $\Delta t=10^{-4}$, the time histories of lift and drag coefficients for two angles of attack are presented in Figure 5: at $14^{\circ}$ which is just at the start of stall, and at $16^{\circ}$ which is beyond the stall point (but not deep stall). The maximum deflection for the dynamically morphing flap is achieved after $t=1 \mathrm{~s}$. For both cases, the dynamic lift rises with time as the flap is dynamically morphed, reaching maximum lift values shortly before the maximum flap deflection is reached. Beyond maximum flap deflection, however, the lift coefficient behaviour is markedly different for the two angles of attack.

At an $\mathrm{AoA}=14^{\circ}$, it is observed that a recirculation zone, or separation bubble (figure not shown) resides on the upper surface of the airfoil near its trailing edge, and grows in size as the flap is dynamically morphed, reaching maximum size at maximum deflection but without any vortex shedding. The drag coefficient time history at AoA $=14^{\circ}$ is similar to the lift coefficient's time history. On the other hand, at AoA $=16^{\circ}$ the lift coefficient drops sharply beyond maximum deflection. Afterward, the lift coefficient settles down into a regular, oscillating time history with two vortex shedding patterns: the first with a period and Strouhal number of $2.4 s$ and 0.018 respectively, the second with a period of $0.05 s$ and a Strouhal number of 0.58 respectively, all while exhibiting small scale oscillations observed at the peaks of each cycle.

Figure 6 gives an in-depth visualisation of the flow evolution for the $16^{\circ}$ AoA. During the dynamic morphing, the separation bubble present on the trailing edge slowly extends to cover most of the airfoil upper surface at $t=1 \mathrm{~s}$ then it undergoes small-scale oscillations without complete separation. However, a small separation bubble is also observed 
around the trailing edge; this bubble grows steadily till it bursts, forming a vortex shedding pattern, which could explain the sudden drop in lift and surge in drag observed around $t=1.5 \mathrm{~s}$. Nevertheless, the bubble forming at the trailing edge afterwards is smaller than the initial one, giving weaker shedding which, again, may explain the lower amplitude of the lift and drag oscillations after the initial transient period. The drag exhibits similar behaviour but notably the drag peak always coincides with minimum lift; this is synonymous with vortex shedding. Compared to the baseline NACA 0012 , the dynamic trailing edge flap seems to destabilize the steady bubble residing on the airfoil upper surface, causing more unsteadiness in the flow.

\section{Conclusion}

Aerodynamic performance analysis of the NACA 0012 airfoil with a morphing trailing edge flap was performed using commercial CFD solver Fluent, by means of a modified parametrisation method implemented with a user defined function (UDF). The simulation of the morphing flap was facilitated with the use of dynamic meshing. Steady CFD results for the deformed mesh were compared with a re-generated mesh to assess the accuracy of the dynamic meshing scheme, followed by a comparative study between a morphed and a flapped airfoil. Finally, an unsteady morphing airfoil investigation was carried out for a dynamic flap and results for two angles of attack near the stall were presented and discussed.

The following conclusions can be drawn:

- The modified unsteady parametrisation method worked well as expected and the deformed mesh gave results similar to the re-generated mesh with small discrepancies of less than $1 \%$, confirming the validity of the geometry and mesh deformation scheme.

- Steady state CFD results obtained from Fluent gave an average of 6\% higher lift and drag coefficients compared with OpenFOAM. Both Fluent and OpenFOAM over-predicted the drag coefficient compared with XFOIL.

- For the same maximum deflection (5\% chord), the morphing airfoil produced higher lift in comparison to a flapped airfoil, but at the expense of a drag penalty. An average increase in aerodynamic efficiency $C_{L} / C_{D}$ of $6.5 \%$ is observed.

- The morphed airfoil reaches a peak aerodynamic efficiency at $8^{\circ}$ angle of attack about $13 \%$ higher than the flapped one, after this angle the efficiency decreases till an angle of $13^{\circ}$. Beyond this angle, the flapped airfoil exhibits better aerodynamic performance, and this trend is qualitatively confirmed by $3 \mathrm{D}$ wind tunnel tests.

- Unsteady analysis of a continuously morphing airfoil has been carried out successfully as proof of concept. Obtained results have shown that the lift and drag coefficient evolution in response to structure shape change is non-linear. Lift reaches a peak value by the time the morphing has stopped, and steadily approaches a 'converged' status afterwards for an angle of $14^{\circ}$.

- For $\mathrm{AoA}=16^{\circ}$, increased unsteadiness caused by the dynamic morphing TE flap is observed in the flow, with two distinct low and high frequency vortex shedding patterns. This is an indication of the rich and complex flow physics associated with this process that needs to be investigated thoroughly.

As further work, it will be important to study the effects of morphing frequency on aerodynamic forces in particular investigating if certain motions can delay or suspend unsteady vortex shedding. Investigating the effects of the trailing edge flap length, flap size, the morphing angle and direction will be important. Finally, in order to resolve the unsteady flow physics observed for the dynamic morphing case, the use of higher fidelity CFD methods beyond RANS (e.g. LES) would be required.

\section{REFERENCES}

Ai, Q., Jawahar, H.K. and Azarpeyvand, M. (2016a), "Experimental investigation of aerodynamic performance of airfoils fitted with morphing trailing edges", 54th AIAA Aerospace Sciences Meeting, American Institute of Aeronautics and Astronautics, (AIAA 2016-1563), doi:10.2514/6.2016-1563.

Ai, Q., Azarpeyvand, M., Lachenal, X. and Weaver, P.M. (2016b), “Aerodynamic and aeroacoustics performance of airfoils with morphing structures”, Wind Energy, Vol. 19 No. 7, pp. 1325-1339, doi: 10.1002/we.1900.

ANSYS $^{\circledR}$ Fluent, 17.0, help system, ANSYS FLUENT Theory Guide, ANSYS, Inc.

Barbarino, S., Bilgen, O., Ajaj, R.M., Friswell, M.I. and Inman, D.J. (2011), “A Review of Morphing Aircraft”, Journal of Intelligent Material Systems and Structures, Vol. 22 No. 9, pp. 823-877,doi: 10.1177/1045389X11414084.

Chekkal, I., Cheung, R., Wales, C., Cooper, J.E., Allen, N., Lawson, S., Peace, A.J., Hancock, S., Cook, R., Standen, P. and Carossa, G.M., (2014). "Design of a morphing wing tip", In 22nd AIAA/ASME/AHS Adaptive Structures Conference (p. 1262). doi:10.2514/6.2014-1262.

Dobrzynski, W. (2010), “Almost 40 Years of Airframe Noise Research: What Did We Achieve? - Slide”, Journal of 
Aircraft, Vol. 47 No. 2, pp. 353-367,doi: 10.2514/1.44457.

Drela, M. (1989), "XFOIL: An Analysis and Design System for Low Reynolds Number Airfoils BT - Low Reynolds Number Aerodynamics", in Proceedings of the Conference Notre Dame, Indiana, USA, 5-7 June 1989, in Mueller, T.J. (Ed.), Springer Berlin Heidelberg, pp. 1-12. doi: 10.1007/978-3-642-84010-4_1

Fincham, J., Beaverstock, C. S., Coles, A. B., Parsons, L. L., Ajaj, R. M. and Friswell, M. I. (2014), "Aerodynamic Forces on Morphing Wings During Span Extension", Advanced Aero Concepts, Design, and Operations. Royal Aeronautical Society, Bristol, UK.

Fluent Inc. (2011), “ANSYS Fluent 14.0 UDF manual”, Vol. 15317 No. November, p. 190.

Harris, C.D. (1990), "NASA supercritical airfoils", NASA Technical Paper, No. 2969, pp. 1-76.

Hilbig, H. and Wagner, H., (1984). "Variable wing camber control for civil transport aircraft". In Proceedings of the 15th Congress of the International Council of the Aeronautical Sciences ,pp. 107-112.

Jacobs, E., Ward, K. and Pinkerton, R. (1933), "The characteristics of 78 related airfoil sections from tests in the variabledensity wind tunnel", National Advisory Committee for Aeronautics, pp. 299-354. doi: 10.1017/CBO9781107415324.004.

Jasak, H., Jemcov, A. and Tukovic, Z. (2007), "OpenFOAM : A C ++ Library for Complex Physics Simulations", International Workshop on Coupled Methods in Numerical Dynamics, Vol. m, IUC Dubrovnik, Croatia, pp. 120.

Lachenal, X., Daynes, S. and Weaver, P.M. (2013), "Review of morphing concepts and materials for wind turbine blade applications", Wind Energy, Vol. 16 No. 2, pp. 283-307.doi: 10.1002/we.531.

Lyu, Z. and Martins, J.R.R.A. (2015), "Aerodynamic Shape Optimization of an Adaptive Morphing Trailing-Edge Wing", Journal of Aircraft, American Institute of Aeronautics and Astronautics, Vol. 52 No. 6, pp. 1951-1970. doi: 10.2514/1.C033116

Molinari, G., Quack, M., Dmitriev, V., Morari, M., Jenny, P. and Ermanni, P. (2011), "Aero-Structural Optimization of Morphing Airfoils for Adaptive Wings", Journal of Intelligent Material Systems and Structures, Vol. 22 No. 10, pp. 1075-1089. doi: 10.1177/1045389X11414089.

Obradovic, B. and Subbarao, K. (2012), "Modeling and Simulation of Morphing Wing Aircraft", Morphing Aerospace Vehicles and Structures, John Wiley \& Sons, Ltd, pp. 87-125. doi: 10.1002/9781119964032.ch5.

Rausch, R.D., Yang, H.T.Y. and Batina, J.T. (1989), "Euler flutter analysis of airfoils using unstructured dynamic meshes", Journal of Aircraft, American Institute of Aeronautics and Astronautics, Vol. 27 No. 5, pp. 436-443. doi: $10.2514 / 3.25295$.

Troldborg, N 2005, "Computational study of the Risø-B1-18 airfoil with a hinged flap providing variable trailing edge geometry", Wind Engineering, vol 29, no. 2, pp. 89-113.

Urnes, J. and Nguyen, N. (2013), "A Mission Adaptive Variable Camber Flap Control System to Optimize High Lift and Cruise Lift to Drag Ratios of Future N+3 Transport Aircraft", AIAA-2013-214, Aerospace Sciences Meetings, pp. 1-24. doi: 10.2514/6.2013-214.

Wang, Z. and Przekwas, A. (1994), "Unsteady flow computation using moving grid with mesh enrichment", 32nd Aerospace Sciences Meeting and Exhibit, American Institute of Aeronautics and Astronautics. doi: 10.2514/6.1994-285.

Wolff, T., Ernst, B. and Seume, J.R., (2014). "Aerodynamic behaviour of an airfoil with morphing trailing edge for wind turbine applications". Journal of Physics: Conference Series, Vol. 524, No. 1, pp. 12018. ISSN: 1742-6596.

Woods, B. K. S., Fincham, J.H. and Friswell, M.I., (2014a), July. Aerodynamic modelling of the fish bone active camber morphing concept. In Proceedings of the RAeS Applied Aerodynamics Conference, Bristol, UK (Vol. 2224).

Woods, B. K. S., Bilgen, O. and Friswell, M. I. (2014b) 'Wind tunnel testing of the fish bone active camber morphing concept', Journal of Intelligent Material Systems and Structures. SAGE Publications, 25(7), pp. 772-785. doi: 10.1177/1045389X14521700.

Woods, B. K. S., Parsons, L., Coles, A. B., Fincham, J. H. S. and Friswell, M. I. (2016) 'Morphing elastically lofted transition for active camber control surfaces', Aerospace Science and Technology. Elsevier Masson SAS, 55, pp. 439-448. doi: 10.1016/j.ast.2016.06.017. 


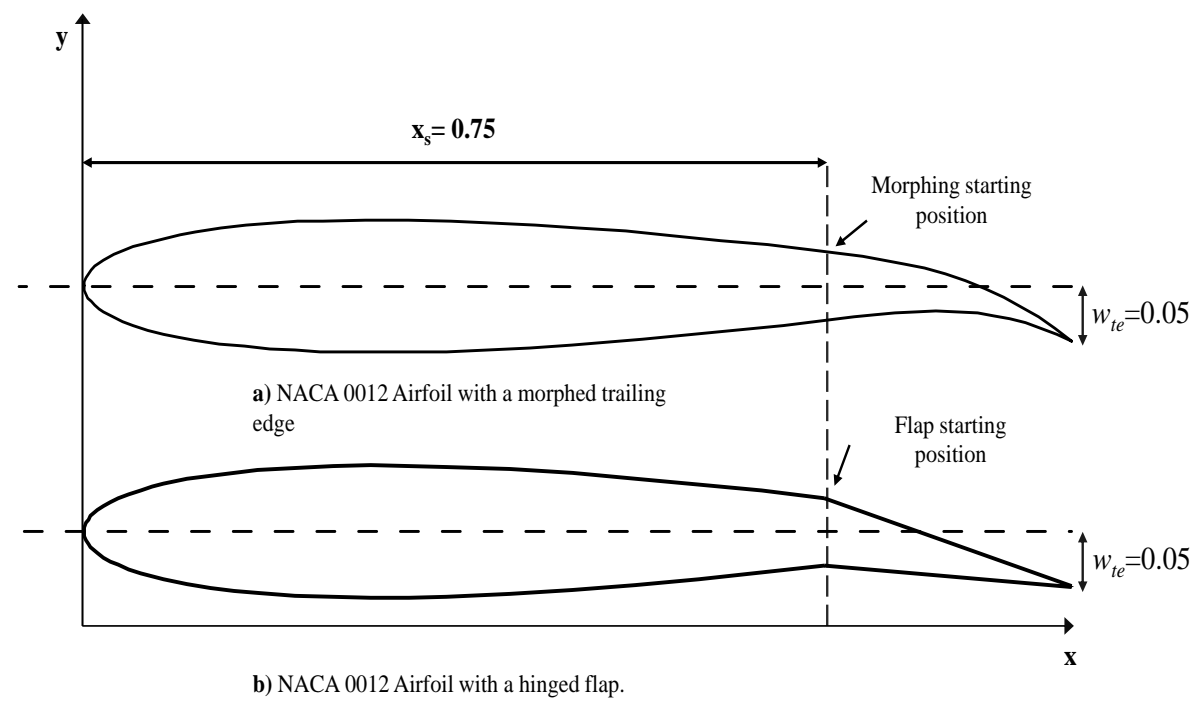

Figure 1. NACA 0012 airfoil with a) morphed and b) hinged flap definitions.

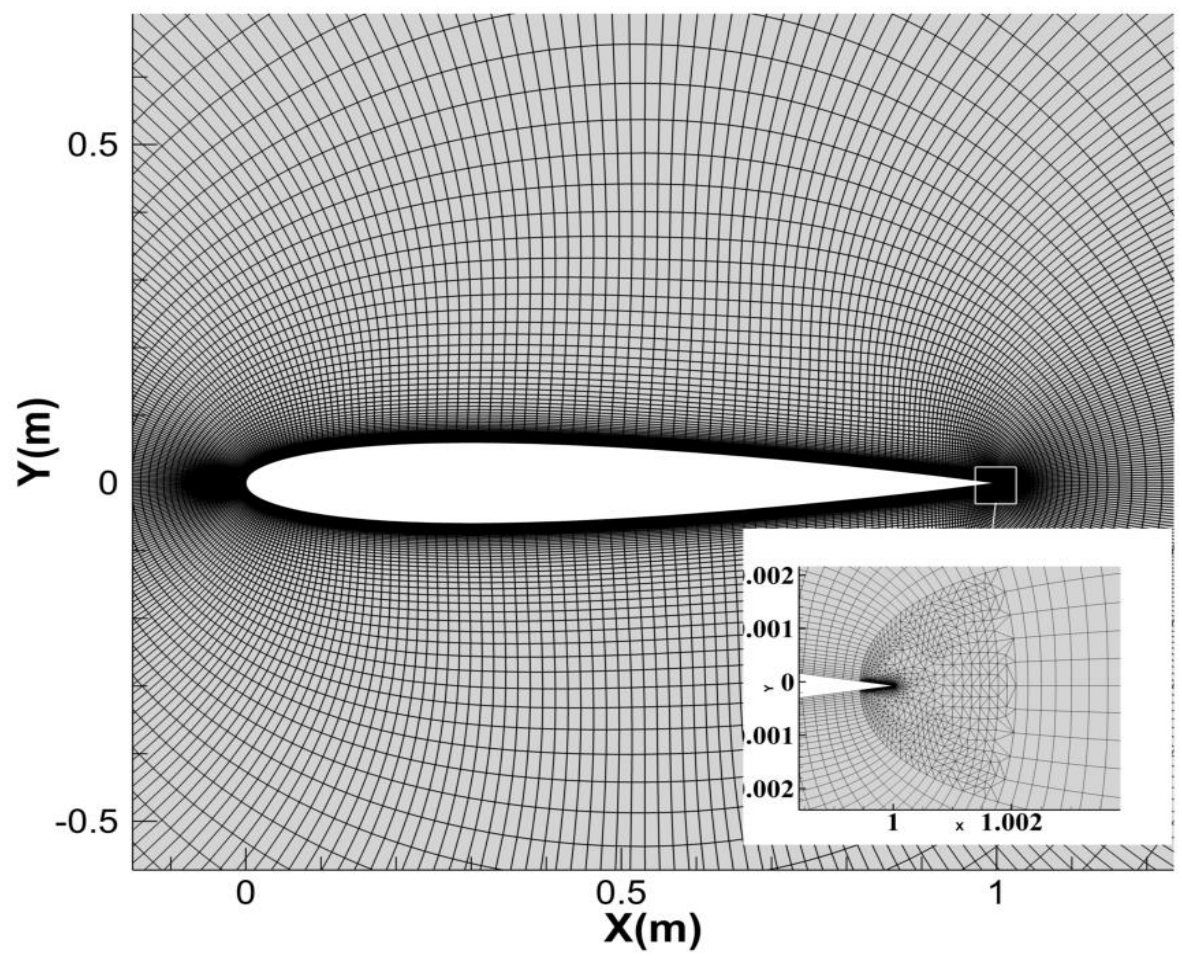

Figure 2a. NACA 0012 mesh with a close up of the triangular patch around the morphing part for an un-deflected case. 


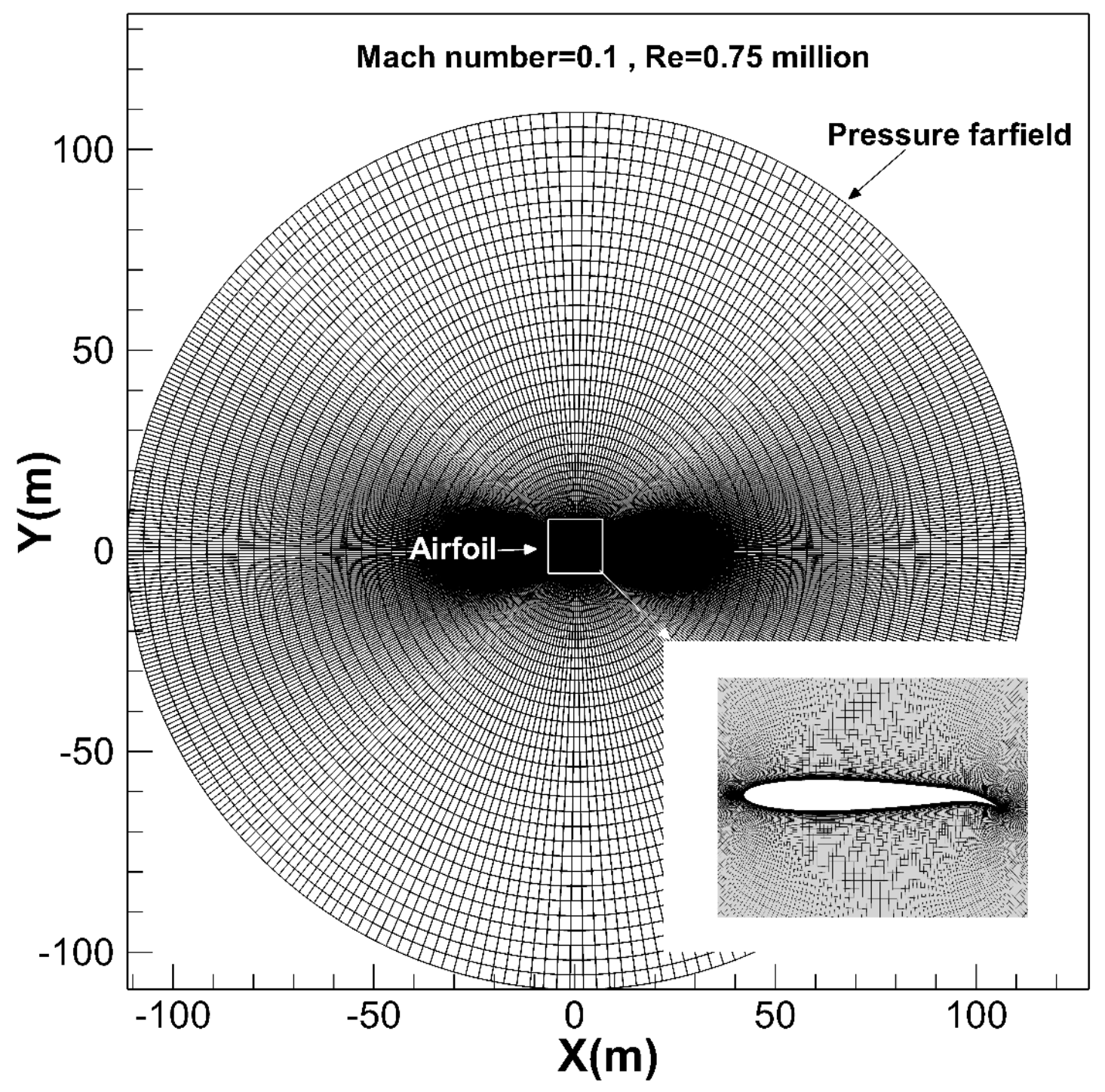

Figure 2b. O-grid type mesh used for the simulations with a close up of the deformed airfoil. 


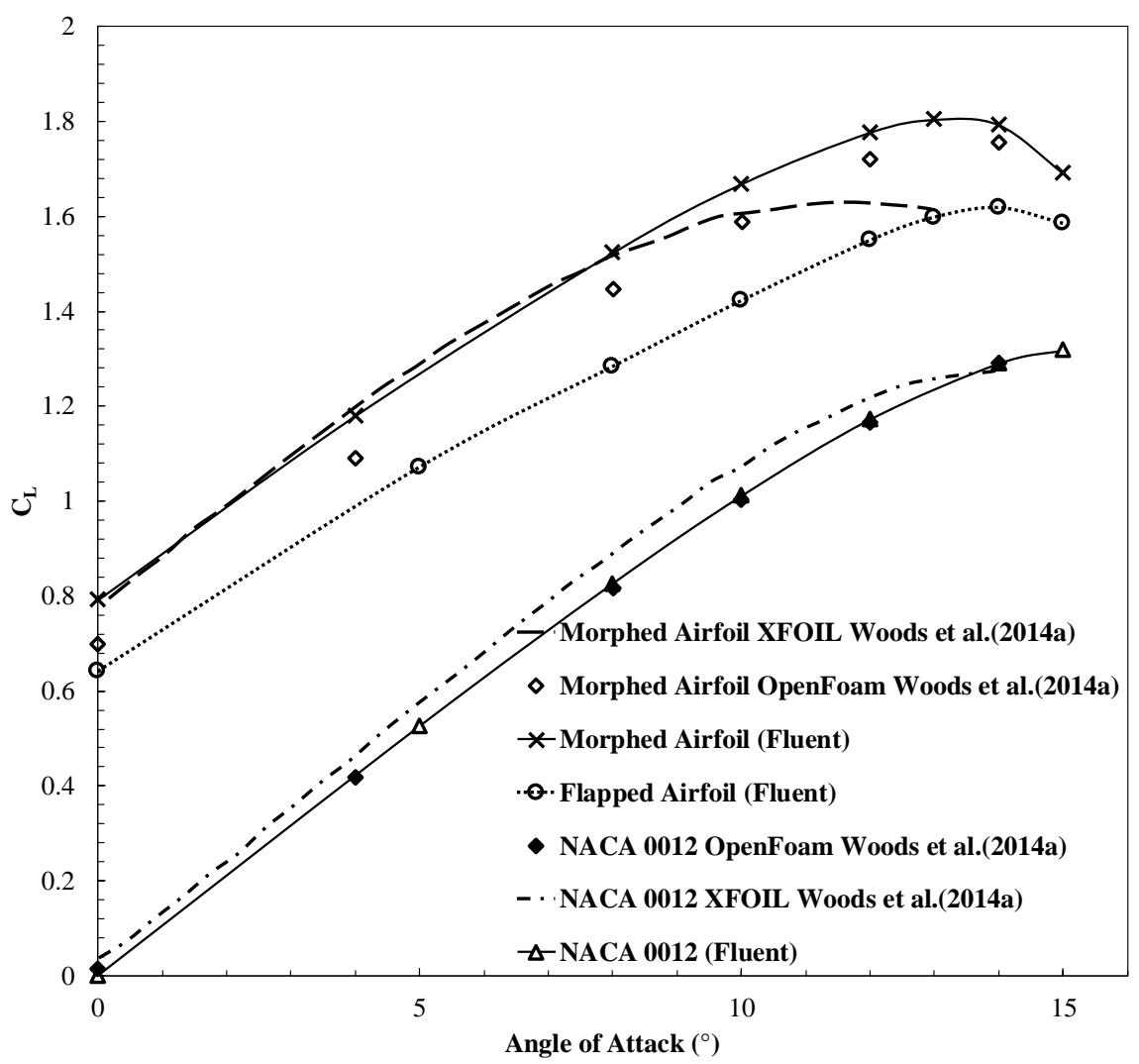

3a) Lift coefficient.

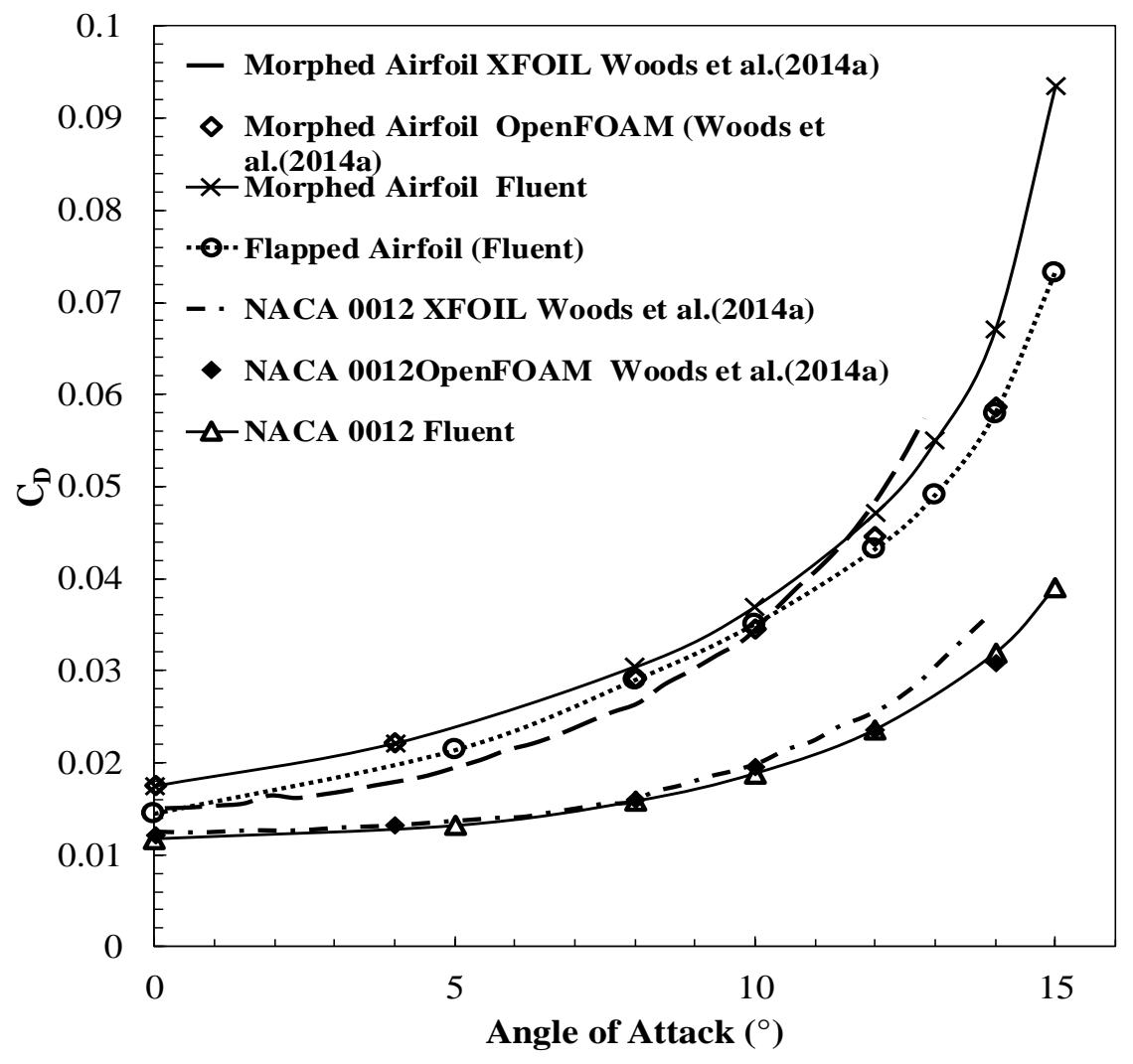

3b) Drag coefficient. 


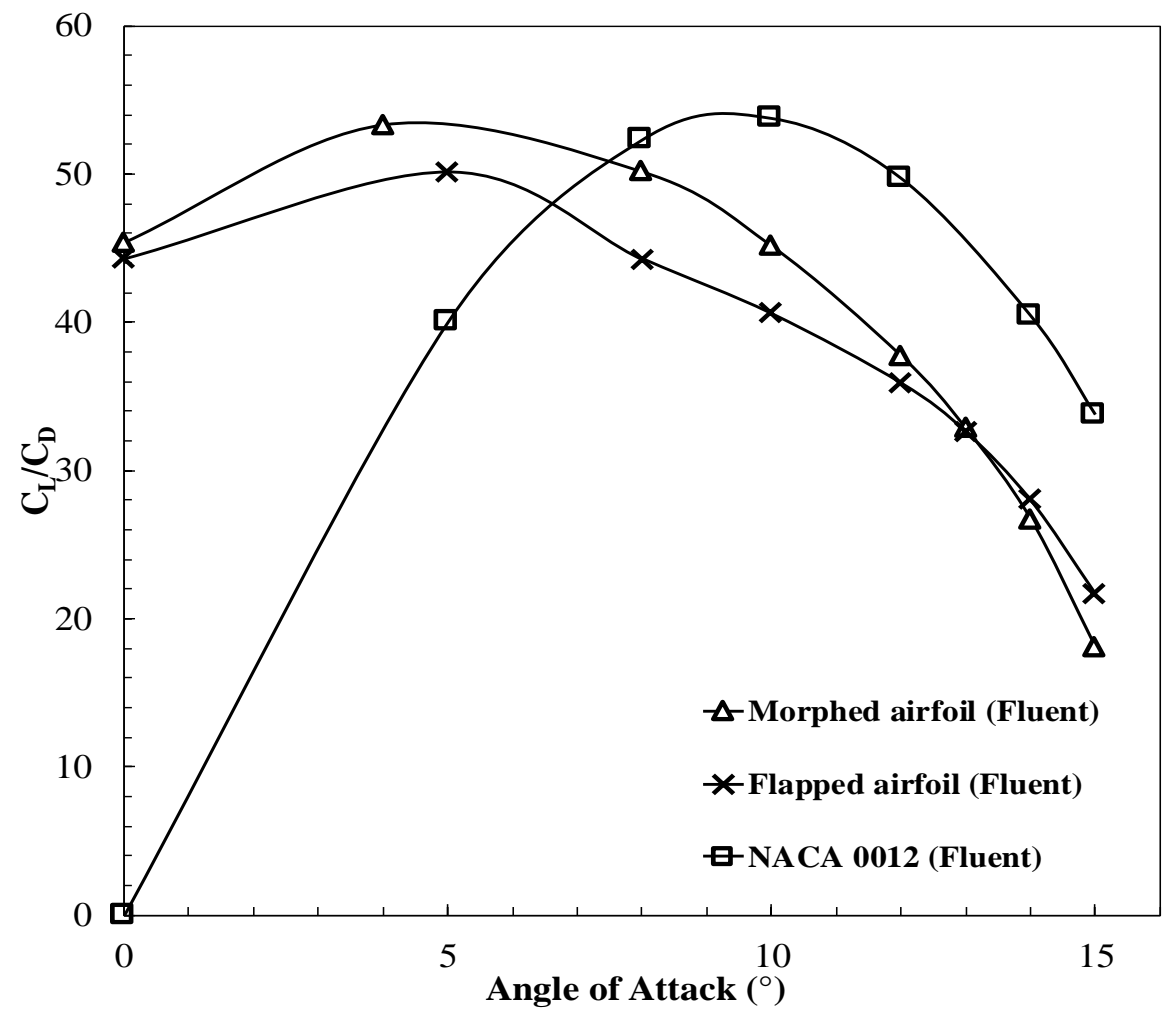

3c) Aerodynamic efficiency $C_{L} / C_{D}$

Figure 3. Validation results for baseline NACA 0012, morphed and flapped airfoil compared with OpenFOAM and XFOIL results of Woods et al. (2014a).

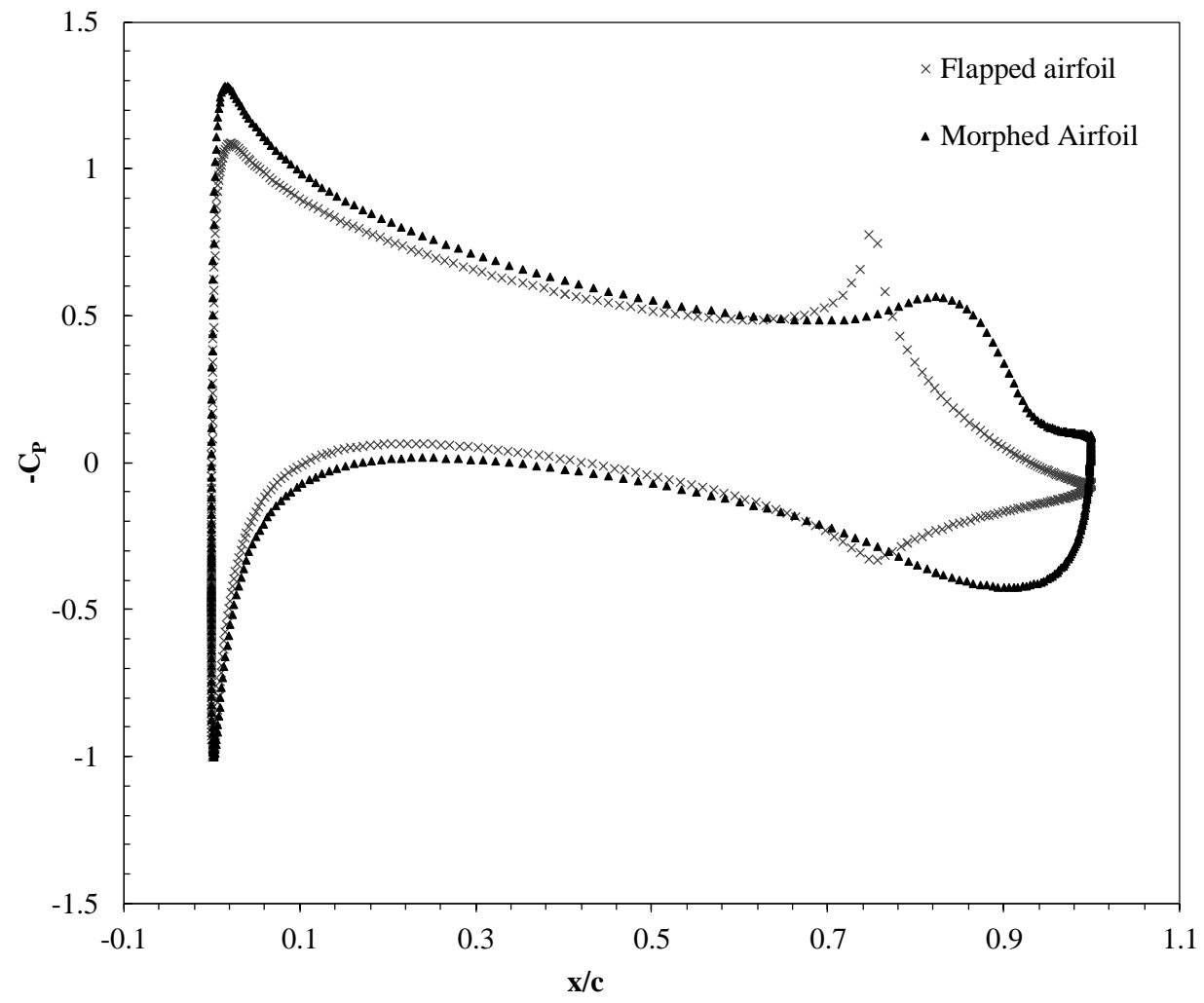

4a) $\mathrm{AoA}=0^{\circ}$ 


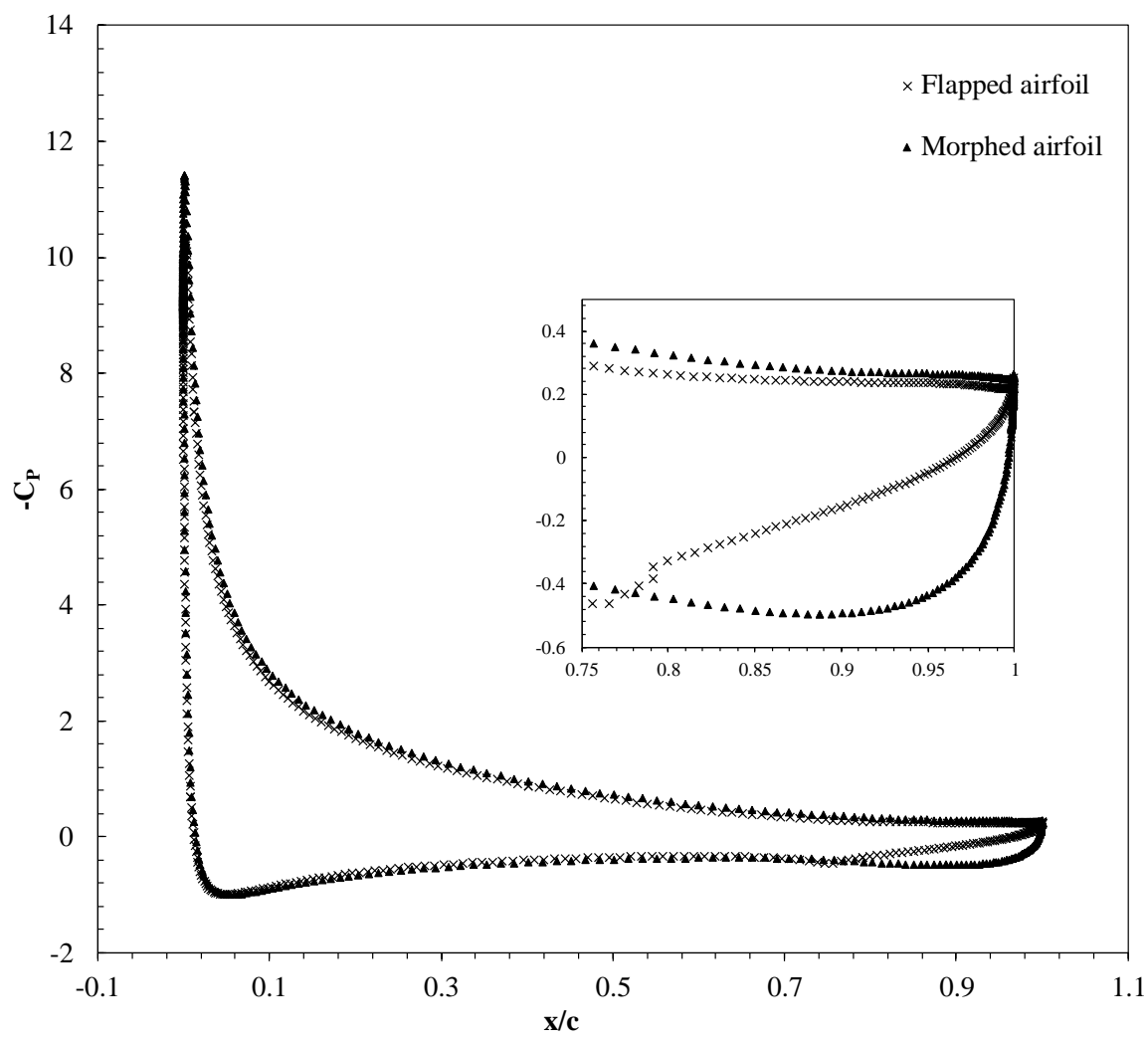

4b) $\mathrm{AoA}=8^{\circ}$

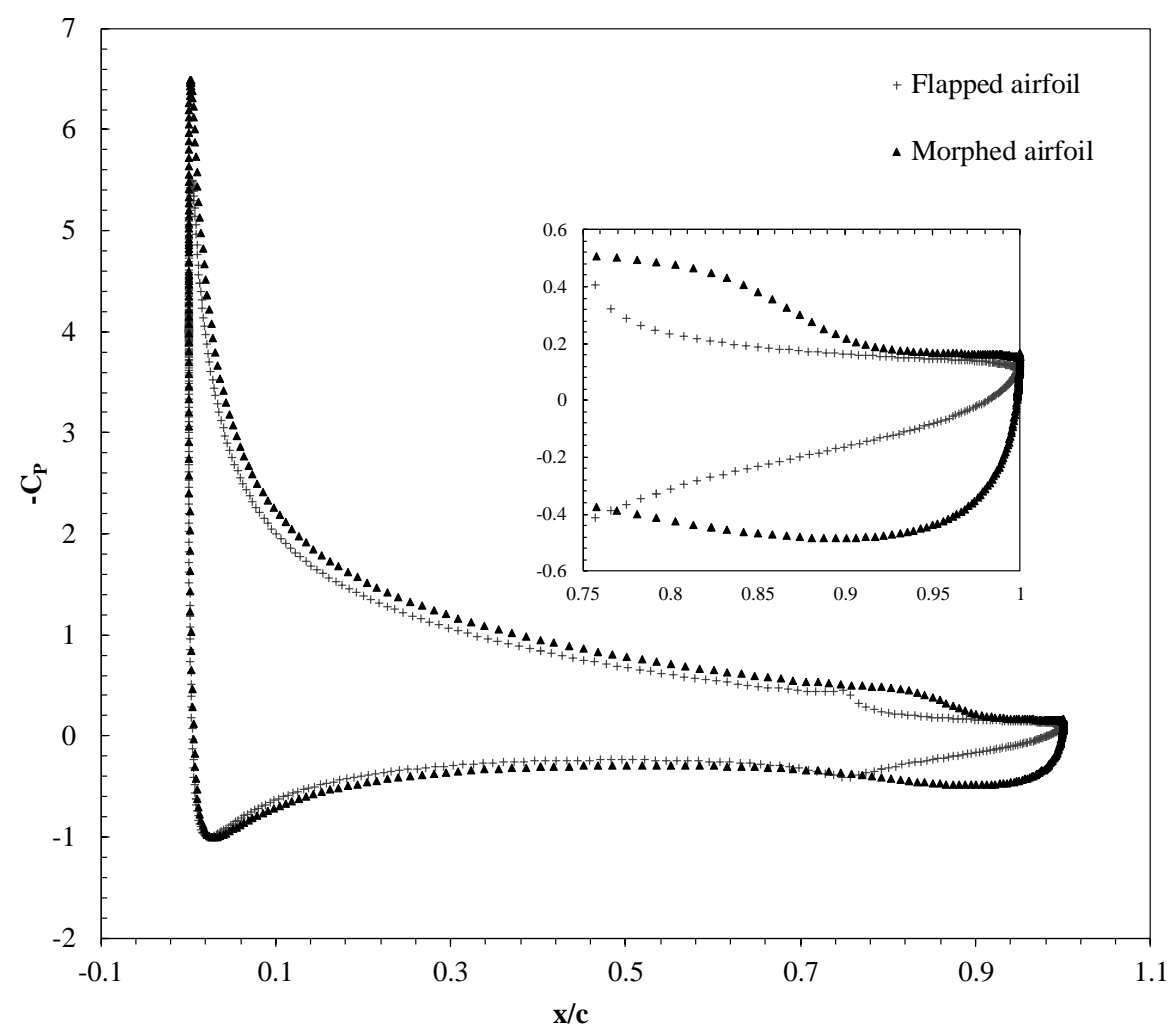

4c) $\mathrm{AoA}=14^{\circ}$

Figure 4. Pressure coefficient $C_{P}$ comparisons between the morphed and flapped airfoils at different angles of attack. 


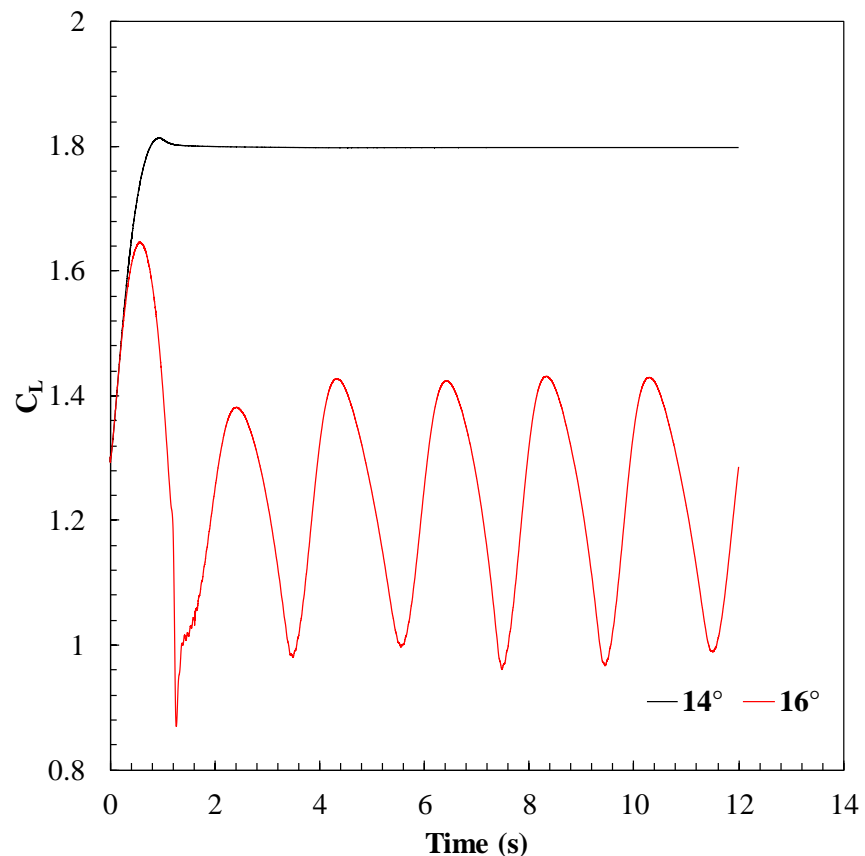

a) Lift coefficient

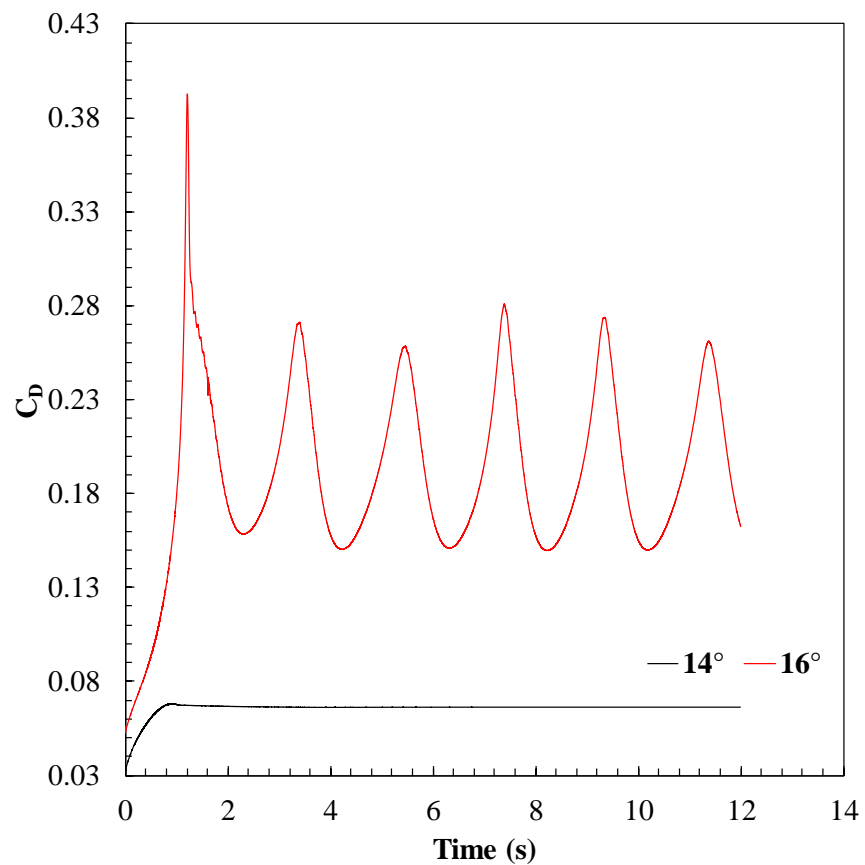

b) Drag coefficient

Figure 5. Time histories of unsteady a) lift coefficient and b) drag coefficient at $14^{\circ}$ and $16^{\circ} \mathrm{AoA}, \Delta t=10^{-4} \mathrm{~s}$. 

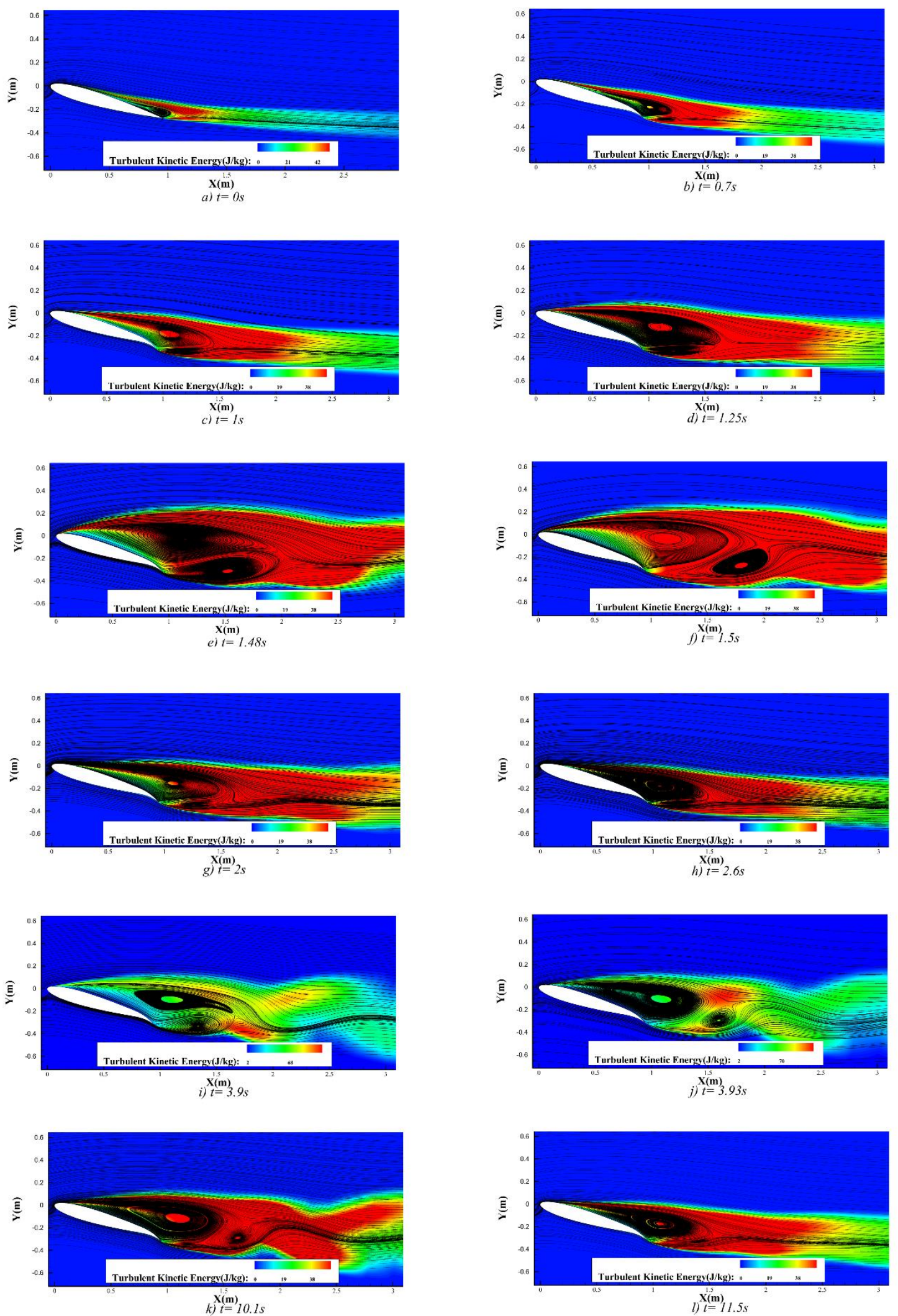

Figure 6. Time history of TKE and flow streamlines around the NACA 0012 airfoil with a dynamic morphing flap, at $A o A=16^{\circ}$. 